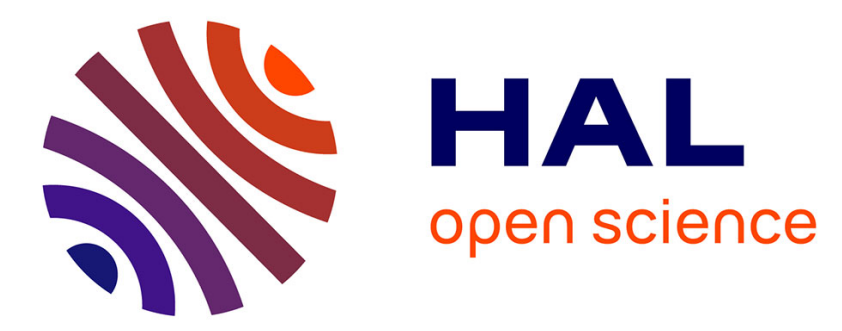

\title{
A New Extension of Desired Compensation Adaptive Control and its Real-Time Application to Redundantly Actuated PKMs
}

\author{
Moussab Bennehar, Ahmed Chemori, François Pierrot
}

\section{- To cite this version:}

Moussab Bennehar, Ahmed Chemori, François Pierrot. A New Extension of Desired Compensation Adaptive Control and its Real-Time Application to Redundantly Actuated PKMs. IROS: Intelligent RObots and Systems, Sep 2014, Chicago, United States. pp.1670-1675, 10.1109/IROS.2014.6942779 . hal-01070862

\section{HAL Id: hal-01070862 \\ https://hal.science/hal-01070862}

Submitted on 29 Oct 2014

HAL is a multi-disciplinary open access archive for the deposit and dissemination of scientific research documents, whether they are published or not. The documents may come from teaching and research institutions in France or abroad, or from public or private research centers.
L'archive ouverte pluridisciplinaire HAL, est destinée au dépôt et à la diffusion de documents scientifiques de niveau recherche, publiés ou non, émanant des établissements d'enseignement et de recherche français ou étrangers, des laboratoires publics ou privés. 


\title{
A New Extension of Desired Compensation Adaptive Control and its Real-Time Application to Redundantly Actuated PKMs
}

\author{
M. Bennehar*, A. Chemori* and F. Pierrot*
}

\begin{abstract}
In this paper, a new control scheme based on the desired compensation adaptive control strategy for mechanical manipulators is developed. In order to estimate the unknown parameters, the adaptation law is formulated based on the inverse dynamic model and the desired trajectories instead of the actual ones. To further improve the tracking performance and the disturbance rejection ability of the original controller, the static feedback gains are replaced by nonlinear varying ones. The computed control inputs are then projected using a kinematics based projector in order to remove the internal efforts in redundantly actuated parallel kinematic manipulators that may damage the mechanical structure of the manipulator. To demonstrate its effectiveness, the proposed controller is validated through real-time experiments on Dual-V; a 3-DOFs redundantly actuated parallel kinematic manipulator. The obtained results show that the proposed controller outperforms the original one in terms of tracking errors and energy consumption.
\end{abstract}

\section{INTRODUCTION}

Parallel Kinematic Manipulators (PKMs) have known a growing interest in the last few decades, both in scientific and industrial communities. These mechanisms feature many advantageous characteristics over their serial counterparts; they are faster, lighter, stiffer and more accurate [1]. Moreover, actuation redundancy further improves their performance by canceling singularities and homogenizing the performance throughout the workspace [2]. Nevertheless, PKMs exhibit many drawbacks and difficulties that narrow their industrial expansion and limit their applications. Indeed, the inherent closed-chains structure and the associated kinematic constraints significantly reduce the usable workspace and give raise to complex nonlinear dynamics [3]. As a result, the control design turns into a very challenging task.

In the literature, many control strategies for PKMs have been proposed [4]-[6]. They can be classified into two main categories, decentralized and dynamic strategies. In decentralized strategies, the control task is reduced into controlling each actuator separately by a linear single axis controller such as the Proportional Derivative (PD) [7] and the Nonlinear PD (NPD) [8]. This class of controllers is the most widespread in industry thanks to its simplicity and efficiency. However, in decentralized control strategies, neither the nonlinear coupled dynamics nor the closedchains constraints are taken into account. Consequently, their performance deteriorates especially in high speed tasks due

*LIRMM, Université Montpellier 2 - CNRS, 161 rue Ada, 34392 Montpellier, France. Email: \{bennehar, chemori, pierrot\}alirmm.fr

This research was supported by the French National Research Agency, within the project ANR-ARROW. to the non trivial nonlinearities. Though PKMs are mainly designed to achieve high accelerations and high accuracy, decentralized controllers fail into achieving this goal. On the other hand, dynamic controllers mainly rely on the dynamic model of the manipulator in order to compensate the nonlinearities. Therefore, dynamic controllers allow to achieve higher accelerations and maintain high accuracy. The Computed Torque (CT) [9] and the Augmented PD (APD) [10] controllers belong to this class. The main drawback of such controllers is the need of an accurate dynamic model of the manipulator so that the nonlinearities are properly compensated.

To overcome the problem of inaccuracies and uncertainties, adaptive control was introduced to mechanical manipulators [11]. Thanks to its ability to adapt to environment and load changes, adaptive control seems to be a suitable choice to cope with the aforementioned issues. Though this advanced control strategy has been extensively investigated in serial manipulators [11], [12], it has not gained the same interest in PKMs. In [13], the backstepping technique has been employed to design an adaptive controller for the control of a 2-DOFs planar PKM. The proposed controller was successfully implemented in real-time and showed its superiority in comparison with conventional controllers but it was only developed for set-point trajectories. In [14], a task-space adaptive controller was proposed to control a 2DOFs redundantly actuated PKM (RA-PKM). The gradient descent algorithm was used to minimize a performance index based on the tracking errors. Stability of the controller was demonstrated using Lyapunov theory and the conducted realtime experiments showed that the proposed controller leads to better performances than the APD. Unfortunately, the convergence of the estimated parameters was not illustrated. The Desired Compensation Adaptive Law (DCAL) [15] was proposed in [16] to control the Hexaglide, a 6-DOFs PKM intended to be used for high speed milling. The suggested controller allowed to estimate all the inertial and friction parameters of Hexaglide in real-time. Nevertheless, the accuracy of such a controller can be further improved by careful design of the feedback gains.

In this paper we propose a novel extension to the DCAL controller in order to enhance its performance. It was heuristically shown in [17] and [18] that using variable nonlinear feedback gains leads to superior tracking performance in comparison with static ones and endow the controller with a better disturbance rejection. Consequently, we propose herein to amend the DCAL controller by replacing the static feedback gains by nonlinear ones. The motivation behind 
this proposition is to improve the tracking performance of the DCAL controller while preserving the advantages of adaptive control. For comparison purpose, original DCAL and its proposed extended version are implemented in realtime on Dual-V [19]; a planar 3-DOFs RA-PKM, in order to demonstrate the outcomes of the proposed extension.

This paper is organized as follows. In section 2, the dynamic modeling of the Dual-V is presented. Section 3 is devoted to the control design description. In section 4, we experimentally implement both controllers on the Dual$\mathrm{V}$ and compare their performance. Finally, in section 5, we draw conclusions about the proposed approach as well as perspectives and future work.

\section{DUAL-V: A PLANAR 4-RRR REDUNDANTLY ACTUATED PARALLEL ROBOT}

Dual-V is a 3-DOFs planar RA-PKM developed within a collaboration between LIRMM (France) and the University of Twente (Netherlands). It belongs to the 4-RRR family (where $\mathrm{R}$ stands for revolute joint) in which every RRR chain is composed of an actuator, a crank and a coupler. The arrangement of the chains allows one rotation about the $\mathrm{z}$ axis and two translations on the xy-plane for its end effector (a traveling plate).

The displacements of the traveling plate of Dual-V are given with respect to a reference frame placed in the center of the rectangle formed by its four actuators. The operational coordinates of the traveling plate are described by the vector $X=[x, y, \theta]^{T} \in \mathbb{R}^{3}$ where the pair $(x, y)$ stands for the position in the horizontal plane and $\theta$ for the orientation with respect to the $\mathrm{z}$-axis, while the vector $\dot{X}=[\dot{x}, \dot{y}, \dot{\theta}]^{T} \in \mathbb{R}^{3}$ denotes the corresponding operational velocities. The actuated joint positions are expressed by $q=$ $\left[q_{1}, q_{2}, q_{3}, q_{4}\right]^{T} \in \mathbb{R}^{4}$ and their corresponding velocities by the vector $\dot{q}=\left[\dot{q}_{1}, \dot{q}_{2}, \dot{q}_{3}, \dot{q}_{4}\right]^{T} \in \mathbb{R}^{4}$. One advantageous feature of the Dual-V robot relies in its having of a closed form analytical expressions for the forward and inverse kinematic problems [19]. Therefore, the transformation from operational to joint space (and vice versa) is straightforward and does not require extensive numerical computations. The mapping from operational to joint velocities is achieved by means of the inverse Jacobian matrix $J_{m}(q) \in \mathbb{R}^{4 \times 3}$ such as

$$
\dot{q}=J_{m} \dot{X}
$$

The torques produced by the four actuators are given by $\Gamma \in \mathbb{R}^{4}$. To establish the inverse dynamic model of the robot, the method proposed in [2] was extended to include the dynamics of the couplers. Indeed, the Dual-V robot differs from the Delta-like robots by having forearms (couplers) of significant masses, therefore, their inertial dynamics cannot be neglected. Consequently, an additional term is added to take into account these dynamics. The full inverse dynamic model of Dual-V is given by

$$
\left(J_{m}^{T}\right)^{*} M_{I} \ddot{X}+M_{I I}\left(\dot{J_{m}} \dot{X}+J_{m} \ddot{X}\right)+\Gamma_{c o m p}=\Gamma
$$

where $\left(J_{m}^{T}\right)^{*}$ denotes the pseudo-inverse of transpose of the jacobian matrix $J_{m}$ and $\ddot{X} \in \mathbb{R}^{3}$ denotes the operational

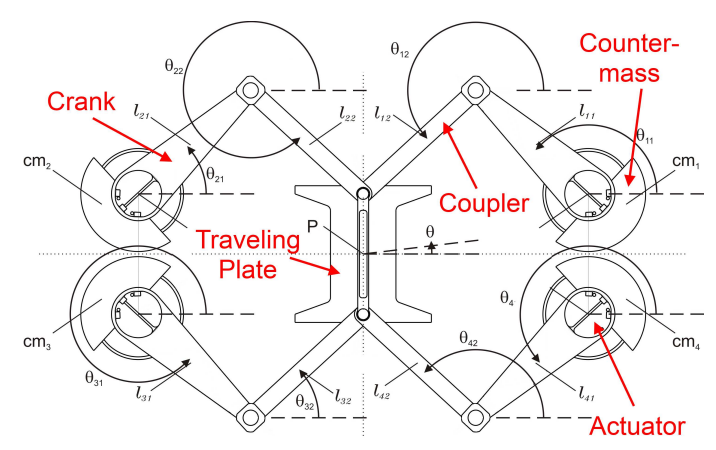

Fig. 1: Illustration of the CAD model of Dual-V robot

acceleration of the traveling plate. $\Gamma_{\text {comp }}=f(X, \dot{X}, \ddot{X}) \in$ $\mathbb{R}^{4}$ is a nonlinear term that accounts for the required torques to compensate for the difference between the modeled and the real couplers inertia. $M_{I} \in \mathbb{R}^{3 \times 3}$ is a diagonal mass matrix of the traveling plate including part of the couplers and $M_{I I} \in \mathbb{R}^{4 \times 4}$ is the mass matrix of the cranks, the counter-masses and part of the couplers as well. The reader may refer to [19] for a more detailed description of Dual-V.

The motivation behind the development of Dual- $\mathrm{V}$ is to produce a high speed dynamically balanced manipulator. This means that when the cranks are equipped with the counter-masses on their rear ends, the shaking forces and moments are significantly reduced. This can be of a considerable interest in some applications where vibrations are unwanted [20].

\section{Control Solution: A New Extension of DCAL}

Consider the dynamics of a n-DOFs manipulator [5]

$$
M(q) \ddot{q}+C(q, \dot{q}) \dot{q}+G(q)=\Gamma
$$

where $M(q) \in \mathbb{R}^{n \times n}$ is the inertia matrix, $C(q, \dot{q}) \in \mathbb{R}^{n \times n}$ is the Coriolis and centrifugal forces matrix and $G(q) \in \mathbb{R}^{n}$ is the gravitational force vector. $q, \dot{q}, \ddot{q} \in \mathbb{R}^{n}$ denote the joint positions, velocities and accelerations respectively. Note that the dynamic model (2) of Dual-V can be rewritten in joint space as (3) using suitable kinematic transformations.

It was shown [11] that the dynamic model (3) can be parametrized into the product of two quantities, a matrix $W(q, \dot{q}, \ddot{q}) \in \mathbb{R}^{n \times p}$ formed by known nonlinear functions of $q, \dot{q}, \ddot{q}$ called the regression matrix, and an unknown parameters vector $\Phi \in \mathbb{R}^{p}$ formed by combinations of the robot parameters such as masses and lengths of the links, thus (3) can be rewritten as

$$
M(q) \ddot{q}+C(q, \dot{q}) \dot{q}+G(q)=W(q, \dot{q}, \ddot{q}) \Phi
$$

Equation (4) is essential in developing any model-based adaptive controller. In what follows, we will first recall the DCAL strategy as it was introduced in [15], then we propose a new extension to improve its performance.

\section{A. Background on DCAL Control}

The main objective of the DCAL controller is to design a controller such that the joint positions $q(t)$ exponentially 
track the desired trajectories $q_{d}(t)$ assuming that the parameters in vector $\Phi$ are unknown or uncertain. Let $e_{p}(t)$ be the error between the actual and desired joint position

$$
e_{p}(t)=q(t)-q_{d}(t)
$$

and let $e_{v}(t)$ be the combined error defined by

$$
e_{v}(t)=\dot{e}_{p}(t)+\lambda_{p} e_{p}(t), \quad \lambda_{p}>0 .
$$

The DCAL control law is expressed as [15]

$$
\begin{aligned}
\Gamma_{D C A L}(t)= & W\left(q_{d}, \dot{q}_{d}, \ddot{q}_{d}\right) \hat{\Phi}-K_{p} e_{p}(t) \\
& -K_{v} e_{v}(t)-f\left(e_{p}, e_{v}\right)
\end{aligned}
$$

where $\hat{\Phi} \in \mathbb{R}^{p}$ is an estimate of the unknown vector $\Phi$ and is adjusted, as proposed in [15], according to the following adaptation law

$$
\dot{\hat{\Phi}}(t)=-K W^{T}\left(q_{d}, \dot{q}_{d}, \ddot{q}_{d}\right) e_{v}(t), \quad K>0 .
$$

$K_{p}$ and $K_{v}$ are positive definite feedback gain matrices and $f(e, \dot{e})=\sigma\left|e_{p}\right|^{2} e_{v}, \quad \sigma>0$ is introduced to compensate for the errors that can emerge from using the desired values instead of the actual ones (the reader may refer to [15] for more details about DCAL).

Notice that in the adaptation law (8), the desired trajectories are used instead of the actual ones. Consequently, the regression matrix $W($.$) can be computed and stored off-line$ which can reduce the computation burden. Furthermore, the adaptation process is more robust against measurement noise since the latter does not affect the desired trajectories.

\section{B. Proposed Extension: A Nonlinear Feedback DCAL}

In a recent work [17], it has been experimentally shown that using nonlinear varying feedback gains instead of constant ones significantly improves the tracking performance of the controller. For instance, the Nonlinear Computed Torque (NCT) [17] and the Nonlinear Augmented PD (NAPD) [18] provided better tracking performance than the conventional CT and APD controllers respectively. A nonlinear PD (NPD) feedback is any feedback term of the form

$$
\Gamma_{N P D}=-K_{p}(.) e_{p}(t)-K_{v}(.) e_{v}(t)
$$

where $K_{p}($.$) and K_{v}($.$) are time varying feedback gains that$ depend on the system state and possibly other variables.

To further improve the tracking performance of DCAL, we propose to modify the control law (7) as follows

$$
\begin{aligned}
\Gamma_{E D C A L}= & W\left(q_{d}, \dot{q}_{d}, \ddot{q}_{d}\right) \hat{\Phi}-K_{p}(.) e_{p}(t) \\
& -K_{v}(.) e_{v}(t)-f\left(e_{p}, e_{v}\right)
\end{aligned}
$$

where $K_{p}($.$) and K_{v}($.$) are nonlinear gains defined as [17]$

$$
\begin{aligned}
& K_{p}\left(e, \alpha_{1}, \delta_{1}\right)=\left\{\begin{array}{cc}
k_{p}|e|^{\alpha_{1}-1}, & |e|>\delta_{1} \\
k_{p} \delta_{1}^{\alpha_{1}-1}, & |e| \leq \delta_{1}
\end{array}\right. \\
& K_{v}\left(\dot{e}, \alpha_{2}, \delta_{2}\right)=\left\{\begin{array}{cc}
k_{v}|\dot{e}|^{\alpha_{2}-1}, & |\dot{e}|>\delta_{2} \\
k_{v} \delta_{2}^{\alpha_{2}-1}, & |\dot{e}| \leq \delta_{2}
\end{array}\right.
\end{aligned}
$$

where $k_{p}, \alpha_{1}, \delta_{1}, k_{v}, \alpha_{2}$ and $\delta_{2}$ are control design parameters to be tuned in order to obtain satisfactory performance. Appropriate choice of the control parameters allows to have large feedback gain for large error and error rate and small gains for small error and error rate. On one hand, the system will track faster the desired trajectories when the error is large thanks to the large gains. On the other hand, the overshot is reduced thanks to the small gains. The adaptation law for the estimated parameters is the same as (8).

For RA-PKMs, the control inputs may contain internal efforts that create prestress in the mechanism. These forces do not create any motion as they are in the null-space of $J_{m}^{T}$ and can damage the robot if they are not considered in the control scheme. The internal efforts can be significantly reduced by projecting the input torques into the range of $J_{m}^{T}$ [3] thanks to the following projector

$$
R_{J_{m}^{T}}=I-N_{J_{m}^{T}}
$$

where $I \in \mathbb{R}^{n \times n}$ is an identity matrix and $N_{J_{m}^{T}}=I-$ $\left(J_{m}^{T}\right)^{*} J_{m}^{T}$ is the projector to the null-space of $J_{m}^{T}$, which results in $R_{J_{m}^{T}}=\left(J_{m}^{T}\right)^{*} J_{m}^{T}$. Hence, the internal efforts can be reduced by projecting the control inputs into the range space of $J_{m}^{T}$ by using the projection matrix $R_{J_{m}^{T}}$ as follows

$$
\Gamma^{*}=R_{J_{m}^{T}} \Gamma_{\text {input }}
$$

where $\Gamma_{\text {input }} \in \mathbb{R}^{n}$ is the torque input generated by any control scheme (i.e DCAL). As from now, the proposed control scheme given by (10) will be referred to as the extended DCAL controller (EDCAL).

\section{Application to the Dual-V Robot}

The dynamic model of Dual-V can be seen as the sum of three separate components thanks to its modular modeling approach [19] as follows

- $\Gamma_{\text {plate }}=\left(J_{m}^{T}\right)^{*} M_{I} \ddot{X}$ is the required actuator torque to move the traveling plate and half the mass of the couplers.

- $\Gamma_{m e c h}=M_{I I}\left(\dot{J_{m}} \dot{X}+J_{m} \ddot{X}\right)$ is the required actuator torque for moving the cranks, the counter-masses and the remaining mass of the couplers.

- $\Gamma_{\text {comp }}=f(X, \dot{X}, \ddot{X})$ is the necessary torque for the compensation of the difference between the modeled couplers' inertias and their CAD values.

Our main motivation to develop an adaptive controller for Dual- $\mathrm{V}$ is to handle load changes when carrying additional masses by means of the traveling plate. This means adjusting the dynamic model according to the variations of the handled object mass. Therefore, thanks to its modular modeling, the adaptation algorithm, without loss of generality, can be reduced to only estimate the dynamic parameters of the traveling plate (i.e. the mass matrix $M_{I}$ ). This a justifiable hypothesis since the additional load will only affect the traveling plate. This approach enables us to estimate the carried weight while taking advantage of the already known steady dynamic parameters of the other links. Therefore, the actual EDCAL control law applied to the robot is given by 


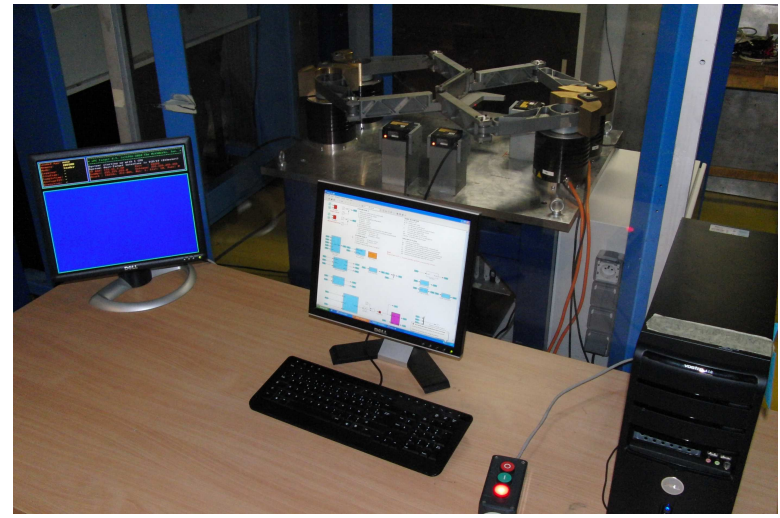

Fig. 2: View of the experimental setup of Dual-V

$$
\begin{aligned}
\Gamma= & R_{J_{m}^{T}}\left[W_{\text {plate }}\left(X_{d}, \ddot{X}_{d}\right) \hat{\Phi}_{\text {plate }}+M_{I I}\left(\dot{J}_{m} \dot{X}_{d}+J_{m} \ddot{X}_{d}\right)+\right. \\
& \Gamma_{\text {comp }}\left(X_{d}, \dot{X}_{d}, \ddot{X}_{d}\right)-K_{p}(.) e_{p}(t) \\
& \left.-K_{v}(.) e_{v}(t)-f\left(e_{p}, e_{v}\right)\right]
\end{aligned}
$$

where $K_{p}($.$) and K_{v}($.$) are defined by (11) and (12)$ respectively. The partial regression matrix $W_{\text {plate }}($.$) is used$ instead of the full regression matrix $W($.$) in (8). It only$ accounts for the dynamics of the traveling plate, a part of the couplers and eventually the additional carried object and is given by

$$
W_{\text {plate }}(X, \ddot{X})=\left(J_{m}^{T}\right)^{*}\left[\begin{array}{cc}
\ddot{x} & 0 \\
\ddot{y} & 0 \\
0 & \ddot{\theta}
\end{array}\right]
$$

The reduced unknown parameters vector $\Phi_{\text {plate }}$ contains only two parameters, the estimated mass $\hat{m}_{11}$ and the estimated inertia $\hat{I}_{11}$ of the travailing plate in addition to a part of the mass of the couplers

$$
\hat{\Phi}_{\text {plate }}=\left[\begin{array}{c}
\hat{m}_{11} \\
\hat{I}_{11}
\end{array}\right] .
$$

\section{VALIDATION OF THE PROPOSED CONTROLLER IN EXPERIMENTS}

\section{A. Experimental Testbed}

To further demonstrate the relevance of the proposed EDCAL controller, real-time experiments have been conducted on Dual-V. Its mechanical structure is composed of several links made with aluminum and mounted on four direct drive actuators from ETEL Motion Technologies, providing each up to $127 \mathrm{Nm}$ torque. The passive joints are made with SKF ball bearings and steel axis. The control loop is designed via Simulink and Real-Time Workshop software, both from

TABLE I: Scenario 1: Tracking performance comparison

\begin{tabular}{|c|c|c|}
\hline & $\mathrm{RMS}_{e_{x y}}[\mathrm{~m}]$ & $\mathrm{RMS}_{e_{\theta}}[\mathrm{deg}]$ \\
\hline DCAL & $4.8173 \times 10^{-4}$ & $4.01 \times 10^{-2}$ \\
\hline EDCAL & $4.1901 \times 10^{-4}$ & $3.63 \times 10^{-2}$ \\
\hline Improvement & $13 \%$ & $9.5 \%$ \\
\hline
\end{tabular}

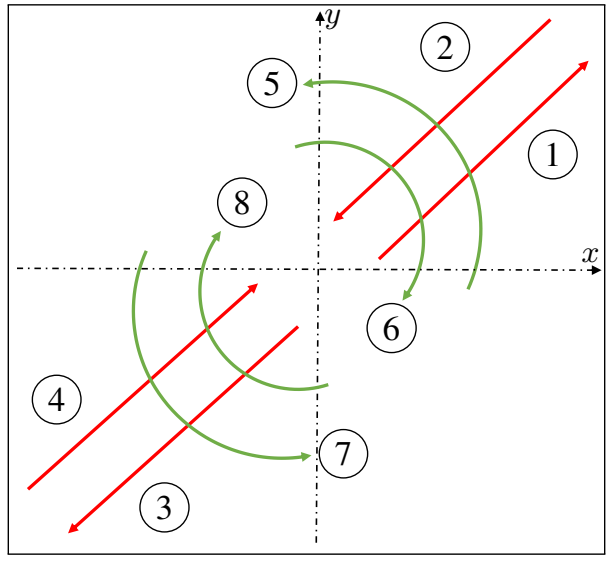

Fig. 3: Illustration of the desired PtP movements to be performed by the robot: center $(0,0)[\mathrm{m}]$, right upper corner $(0.02,0.04)[\mathrm{m}]$, left bottom corner $(-0.02,-0.04)[\mathrm{m}]$, the rotations: $0,+20$ and -20 [deg] anti-clockwise.

Mathworks Inc. The Target PC consists of an industrial computer cadenced at $10 \mathrm{kHz}$ running under $\mathrm{xPC}$ target in real-time. The experimental testbed setup is shown in Fig. 2.

The desired trajectories were generated on-line in Cartesian space using 5 th order polynomials. The desired joint positions and velocities were obtained by solving the inverse kinematics and equation (1) respectively. The actual joint positions are provided by the encoders and the actual Cartesian positions are obtained by solving the forward kinematics problem. The joint velocities were obtained by filtering the joint positions (the actuators are not equipped with velocity sensors). The traveling plate of the Dual-V has to perform Point-to-Point (PtP) trajectories across different positions throughout the workspace. The duration of each PtP trajectory was fixed to 0.25 seconds. Fig. 3 illustrates the set of PtP trajectories performed in one cycle while the complete trajectory contains 10 cycles. Regarding the estimated parameters, a standard mid-point algorithm was implemented to integrate eq. (8).

To evaluate the performance of both controllers, we introduce the following RMS based criteria

$$
\operatorname{RMS}_{e_{x y}}=\sqrt{\operatorname{RMS}\left(e_{x}\right)^{2}+\operatorname{RMS}\left(e_{y}\right)^{2}}
$$

where $e_{x}$ and $e_{y}$ are the Cartesian tracking errors on $x$ and $y$ axes respectively. The tracking error of the rotational movement $e_{\theta}$ is evaluated separately.

\section{B. Real-Time Experimental Results}

To demonstrate the robustness of both controllers to load changes, we experiment two different scenarios; with and

TABLE II: Scenario 2: Tracking performance comparison

\begin{tabular}{|c|c|c|}
\hline & $\mathrm{RMS}_{e_{x y}}[\mathrm{~m}]$ & $\mathrm{RMS}_{e_{\theta}}[\mathrm{deg}]$ \\
\hline DCAL & $4.8726 \times 10^{-4}$ & $4.12 \times 10^{-2}$ \\
\hline EDCAL & $4.2056 \times 10^{-4}$ & $3.74 \times 10^{-2}$ \\
\hline Improvement & $13.6 \%$ & $9.2 \%$ \\
\hline
\end{tabular}



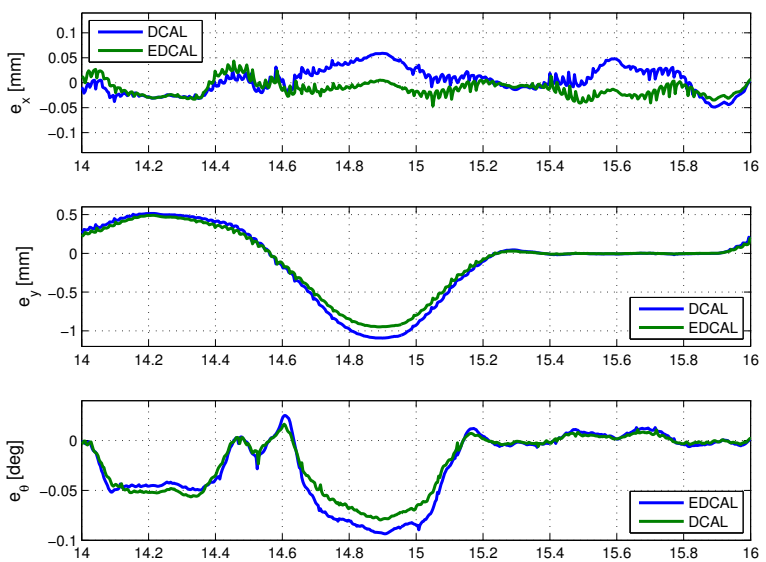

Fig. 4: Scenario 1: Evaluation of Cartesian tracking errors

without a carried payload. The DCAL feedback gains were obtained by trial and error as follows: $K_{p}=35 \times 10^{2}$ and $K_{v}=25$, while the EDCAL feedback gains are tuned using the proposed in [14] leading to the following values $k_{p}=44 \times 10^{3}, \delta_{1}=2 \times 10^{-3}, \alpha_{1}=1.45, k_{v}=75$, $\delta_{2}=2 \times 10^{-2}$ and $\alpha_{2}=1.3$. Both tuning methods have taken into account a torque criterion for the selected gains. The adaptation gain matrix for both controllers is taken as $K=\operatorname{diag}\left\{7.5,75 \times 10^{-2}\right\}$.

1) Scenario 1: without payload: In this scenario, the traveling plate is not carrying any additional mass. The objective of this scenario is to check weather the adaptive algorithm is able to estimate the mass and inertia $\left(\hat{m}_{11}\right.$ and $\hat{I}_{11}$ respectively) and to compare the performance of both controllers. The Cartesian tracking errors are displayed in Fig. 4 for different time spans. It can be clearly seen that the proposed EDCAL controller provides better results than DCAL. Fig. 6 depicts the evolution of the estimated parameters starting from their initial values $[0,0]^{T}$ to their nominal values. We notice however the presence of small oscillations around the nominal values, which is natural due to the phenomena appearing when dealing with a real phys-
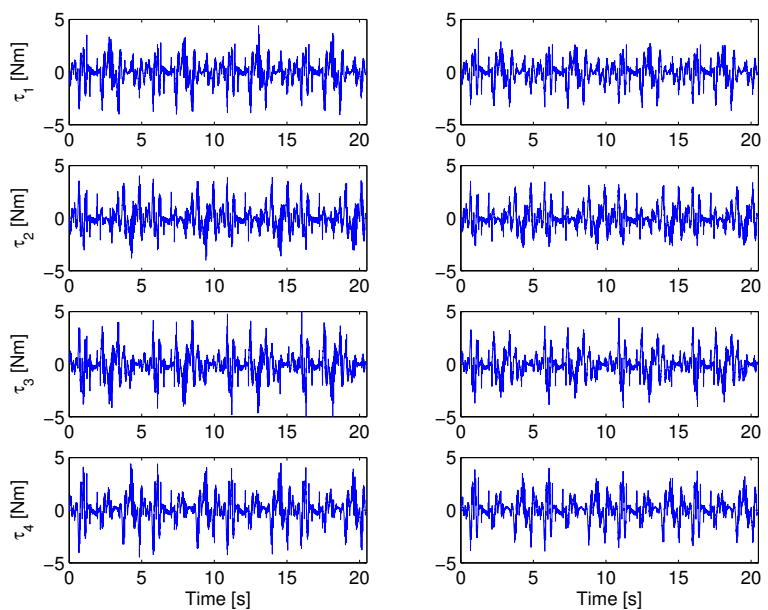

Fig. 5: Scenario 1: Control inputs, left: DCAL, right: EDCAL
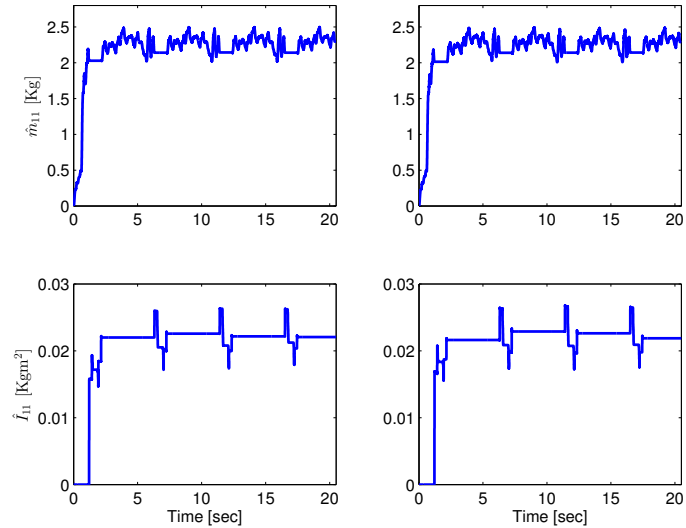

Fig. 6: Scenario 1: evolution of the estimated parameters versus time, left: original DCAL and right: EDCAL.

ical structure (noise, finite encoders resolution, quantization effects, etc.). The generated control inputs are displayed in Fig. 5. We can see that the amplitudes of the torques of the EDCAL controller are slightly smaller than those of DCAL. Table I summarizes and quantifies the tracking performance of both controllers for this scenario using the error tracking RMS-based criteria. One can see that the proposed controller noticeably improves the tracking performance.

2) Scenario 2: with a payload of $6.6 \mathrm{~kg}$ : In this scenario, an additional load is added on top of the traveling plate to check weather the controller adjusts its parameters $\hat{m}_{11}$ and $\hat{I}_{11}$ accordingly. The tracking errors represented in Fig. 7. Again, the proposed EDCAL controller performs better than the original DCAL controller. The difference is more noticeable for large tracking errors. The estimated parameters $\hat{m}_{11}$ and $\hat{I}_{11}$ differ from those of the previous scenario due to the extra load added on the top of the traveling plate. The parameters estimation evolution is depicted in Fig. 9. The adaptation algorithm adjusts the mass parameter from an initial zero value to their new values (cf. Fig. 9), which now includes to the mass of the the added payload. The magnitudes of the generated control inputs of the EDCAL
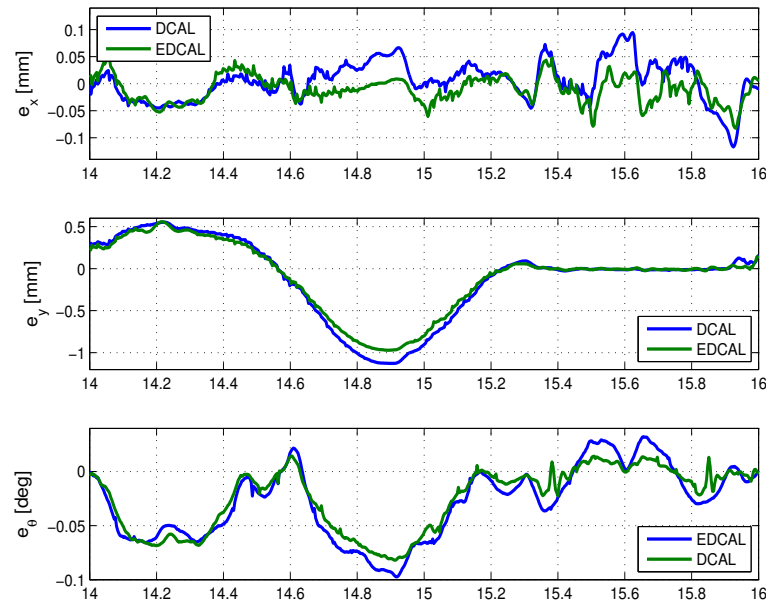

Fig. 7: Scenario 2: Evaluation of Cartesian tracking errors 

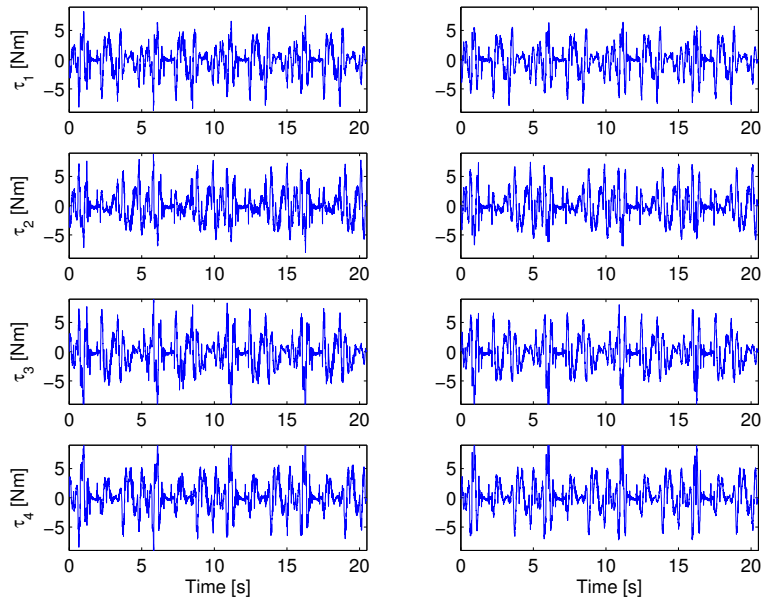

Fig. 8: Scenario 2: Control inputs, left: DCAL, right: EDCAL

controller are slightly smaller than the DCAL one (cf. Fig 8). The performance comparison between both controllers is summarized in Table II. We can see that the proposed extended DCAL provides better results than the original DCAL in terms of tracking accuracy.

Results can be viewed in the video available at the following url: http://youtu.be/M7FZBxmSNqk.

\section{CONCLUSIONS AND FUTURE WORK}

In this paper, an extension to the DCAL control strategy is proposed. The conventional form of the DCAL uses constant linear feedback gains in the error elimination and adaptation process, while it is known that the use of nonlinear variable gains in the control loop can improve its performance. Consequently, we propose to amend the control strategy with variable nonlinear gains function of the error and its derivative. Real-time experiments with two different scenarios have been performed on the Dual-V robot, a redundantly actuated parallel manipulator, in order to demonstrate the superiority of the proposed approach. By comparing the tracking errors of both controllers in the operational space, we conclude that, as it was expected, the nonlinear feedback extension
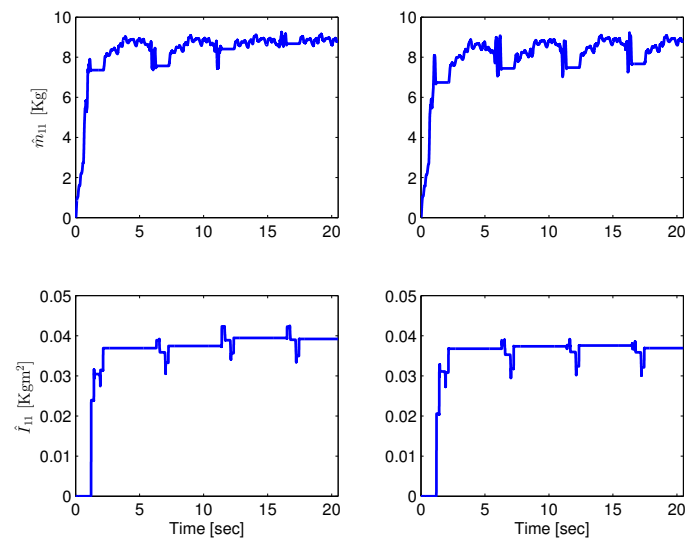

Fig. 9: Scenario 2: Evolution of the estimated parameters versus time, left: DCAL and right: EDCAL significantly improves the performance of the controller. The control scheme can be further improved, in the future, by endowing the adaptation algorithm with known bounds of the uncertain parameters.

\section{REFERENCES}

[1] J. P. Merlet, Parallel Robots, Second Edition. Dordrecht, Netherlands: Springer, 2006.

[2] D. Corbel, M. Gouttefarde, and O. Company, "Towards 100g with pkm. is actuation redundancy a good solution for pick and place?" in Proc. IEEE International Conference on Robotics and Automation (ICRA'10), Anchorage, Alaska, May 2010, pp. 4675-4682.

[3] A. Müller, "Effects of geometric imperfections to the control of redundantly actuated parallel manipulators," in Proc. IEEE International Conference on Robotics and Automation (ICRA'09), Kobe, Japan, May 2009, pp. 1782-1787.

[4] J. F. He, H. Z. Jiang, D. C. Cong, Z. M. Ye, and J. W. Han, "A survey on control of parallel manipulator," Key Engineering Materials, vol. 339, pp. 307-313, May 2007.

[5] H. Cheng, Y.-K. Yiu, and Z. Li, "Dynamics and control of redundantly actuated parallel manipulators," IEEE/ASME Trans. Mechatronics, vol. 8, no. 4, pp. 483-491, Dec. 2003.

[6] F. Paccot, N. Andreff, and P. Martinet, "A review on the dynamic control of parallel kinematic machines: Theory and experiments," The International Journal of Robotics Research, vol. 28, no. 3, pp. 395416, Mar. 2009.

[7] F. L.Lewis, D. M.Dawson, and C. T.Abdallah, Robot Manipulator Control, Theory and Practice. New York, USA: Marcel Dekker, Inc., 2004.

[8] P. R. Ouyang, W. Zhang, and F. Wu, "Nonlinear pd control for trajectory tracking with consideration of the design for control methodology," in Proc. IEEE International Conference on Robotics and Automation (ICRA'02), Washington, USA, May 2002, pp. 4126-4131.

[9] J. Luh, M. Walker, and R. Paul, "Resolved-acceleration control of mechanical manipulators," IEEE Trans. Autom. Control, vol. 25, no. 3, pp. 468-474, Jun. 1980.

[10] D. Koditschek, "Natural motion for robot arms," in Proc. The 23rd IEEE Conference on Decision and Control (CDC'84), Las Vegas, Nevada, USA, Dec. 1984, pp. 733-735.

[11] J. J. Craig, P. Hsu, and S. S. Sastry, "Adaptive control of mechanical manipulators," The International Journal of Robotics Research, vol. 6, no. 2, pp. 16-28, Jun. 1987.

[12] J.-J. E. Slotine, "On the adaptive control of robot manipulators," The International Journal of Robotics Research, vol. 6, no. 3, pp. 49-59, Sep. 1987.

[13] L. Wang, Z. Lu, X. Liu, K. Liu, and D. Zhang, "Adaptive control of a parallel robot via backstepping technique," The International Journal of Systems, Control and Communications, vol. 1, no. 3, pp. 312-324, Apr. 2009.

[14] W. Shang and S. Cong, "Nonlinear adaptive task space control for a 2dof redundantly actuated parallel manipulator," Nonlinear Dynamics, vol. 59, no. 1-2, pp. 61-72, Jan. 2010.

[15] N. Sadegh and R. Horowitz, "Stability and robustness analysis of a class of adaptive controllers for robotic manipulators," The International Journal of Robotics Research, vol. 9, no. 3, pp. 74-92, Jun. 1990.

[16] M. Honegger, A. Codourey, and E. Burdet, "Adaptive control of the hexaglide, a 6 dof parallel manipulator," in Proc. IEEE International Conference on Robotics and Automation (ICRA'97), Albuquerque, New Mexico, USA, Apr. 1997, pp. 543-548.

[17] W. Shang and S. Cong, "Nonlinear computed torque control for a high-speed planar parallel manipulator," Mechatronics, vol. 19, no. 6, pp. 987-992, Sep. 2009.

[18] - Serial and Parallel Robot Manipulators - Kinematics, Dynamics, Control and Optimization, Dr. Serdar Kucuk (Ed.). InTech, 2010, ch. 12.

[19] V. van der Wijk, S. Krut, F. Pierrot, and J. L. Herder, "Design and experimental evaluation of a dynamically balanced redundant planar 4-rrr parallel manipulator," The International Journal of Robotics Research, vol. 32, no. 6, pp. 744-759, May 2013.

[20] Y. Patel and P. George, "Parallel manipulators applications-a survey," Modern Mechanical Engineering, vol. 2, no. 3, pp. 57-64, 2012. 